\title{
Photoluminescence Study of Basal Plane Stacking Faults in ZnO Nanowires
}

\author{
V. Khranovskyy ${ }^{1}$, M. O. Eriksson ${ }^{1}$, G. Z. Radnoczi ${ }^{1,2}$, A. Khalid ${ }^{3}$, H. Zhang ${ }^{3}$, P. O. Holtz ${ }^{1}$, L. \\ Hultman $^{1}$ and R. Yakimova ${ }^{1}$ \\ ${ }^{1}$ Department of Physics, Chemistry and Biology (IFM), Linköping University, \\ 58183 Linköping, Sweden \\ ${ }^{2}$ Research Centre for Natural Sciences, Institute for Technical Physics and Materials \\ Science (MFA), H-1121 Budapest, Konkoly-Thege M. U. 29-33 \\ ${ }^{3}$ School of Physics and Centre for Research on Adaptive Nanostructures and \\ Nanodevices (CRANN), Trinity College Dublin, Dublin 2, Ireland \\ *Corresponding author. \\ e-mail address: volkh@ifm.liu.se \\ tel: $+46-13-282663$ \\ fax: +46-13-137568
}

PACS: 71.55.Gs 73.21.Fg 77.55.hf 78.47.D- 78.47.jd

\begin{abstract}
We have investigated the photoluminescence (PL) of $\mathrm{ZnO}$ nanowires (NWs) containing a high density $\left(\sim 1 \times 10^{6} \mathrm{~cm}^{-1}\right)$ of basal plane stacking faults (BSFs). It was observed that the BSFs result in a specific PL peak at $\sim 3.329 \mathrm{eV}$ along with a donor bound excitonic emission $\left(\mathrm{D}^{0} \mathrm{X}\right)$ peak at $5 \mathrm{~K}$. The observed BSF-related emission is of excitonic type and possesses longer PL lifetime than $\mathrm{D}^{0} \mathrm{X}(\sim 360 \mathrm{ps}$ vs $\sim 70 \mathrm{ps})$. Via comparison of the microstructural and the PL properties of the $\mathrm{ZnO} \mathrm{NWs}$, it is shown that the observed BSF-related emission is due to the formation of crystal phase quantum wells (QWs). This is explained by the fact that BSF in wurtzite (WZ) $\mathrm{ZnO}$ is the thinnest segment of zinc blende $(\mathrm{ZB})$ phase $\mathrm{ZnO}$ inserted in the $\mathrm{WZ}$ matrix, resulting in band alignment of type II due to the conduction and valence band offsets of $\mathrm{ZB}$ with respect to $\mathrm{WZ} \mathrm{ZnO}$. The mechanism of the BSF related PL is suggested to be an indirect exciton transitions due to the recombination of electrons confined in the ZB QWs to holes in the WZ barriers localized near the BSFs.
\end{abstract}

\section{Introduction}

$\mathrm{ZnO}$ is a wide direct band gap semiconductor, which has a number of applications in optoelectronics [1, 2], such as light emitters [3], electron emitters [4] or photo detectors [5]. The 
performance of the devices depends on the structural quality of the material and is strongly influenced by the presence of different defects like impurity, dislocations and/or stacking faults [6]. Defects unintentionally present in the material contribute to its light emission characteristics; low temperature photoluminescence (LT PL) spectra of ZnO typically involve a number of peaks with different origins, which often overlap each other [7]. Defects that serve as non-radiative recombination centers (grain boundaries, threading dislocations, etc.) reduce the light emission efficiency significantly [8]. However, the real challenge is posed by defects that can serve as both non-radiative and radiative centers under certain conditions. One essential example on such a defect is the case of stacking faults (SFs), which are often observed in semiconductors like GaN [9] and $\mathrm{ZnO}$ [10]. SFs come in three main types: i) basal plane SFs (BSFs), ii) prismatic SFs, and iii) partial dislocations-terminating basal plane SFs. BSFs are the most common type of SFs ( 90\%). BSFs may exist both in films and nanostructures of $\mathrm{ZnO}$. Nanorods and nanowires are particularly interesting objects for studies, since BSFs length appear with a same size as the diameter of the nanostructure, penetrating it completely.

Different types of SFs lead to different spectral positions of the PL peaks. However, different types can exist simultaneously, leading to PL peaks that are spectrally separated by a few meV. For $\mathrm{ZnO}$, SFs are difficult to identify in PL spectra, since the spectral locations of the SF related peaks $(3.321-3.333 \mathrm{eV})$ overlap with peaks originating from neutral acceptor bound excitons $\left(\mathrm{A}^{\circ} \mathrm{X}\right)$ and two electron satellite (TES) of the dominating donor bound exciton $\left(\mathrm{D}^{\circ} \mathrm{X}\right)$ emission. This has led to many misinterpretations in the assignment of the peaks in LT PL spectra of $\mathrm{ZnO}$ [10], followed by large data inconsistency in the literature, as summarized by Thonke et al. [11]. At present it is of high scientific interest to identify unambiguously the PL peaks, originating from the $\mathrm{SFs}$ in $\mathrm{ZnO}$ in comparison to conventional $\mathrm{D}^{\circ} \mathrm{X}$ emission. While based on steady-state PL measurements spectral resolving the peaks due to SFs and $\mathrm{D}^{\circ} \mathrm{X}$ can be rather challenging due to above described reasons, time-resolved PL is more appropriate technique, since it enables studying of the dynamics of light emission processes in the semiconductor materials. We have investigated low-temperature and time-resolved PL properties of $\mathrm{ZnO}$ nanowires (NWs), containing a high density of BSFs in comparison to SF free $\mathrm{ZnO}$ nanopillars (NPs) of identical size. From the correlation between the structural and optical properties of $\mathrm{ZnO}$ NWs, the LT PL peak at $\sim 3.329 \mathrm{eV}$ has been attributed to the emission due to available $\mathrm{BSFs}$ in $\mathrm{ZnO}$. 


\section{Experimental}

The $\mathrm{ZnO}$ NWs were prepared by thermal evaporation of a ball milled $\mathrm{ZnO}$ powder at $\sim 1300$ ${ }^{\circ} \mathrm{C}$. Ar flow of $0.41 \mathrm{~min}^{-1}$ was used as a carrier gas to transfer $\mathrm{ZnO}$ vapor from the central hot zone of the furnace to a cooler zone, where Si (100) substrates (n-type) were placed. After one hour, nanowires were formed on the Si substrate, located $17-20 \mathrm{~cm}$ from the vapor source with the temperature estimated to be in range $\sim 250-400{ }^{\circ} \mathrm{C}$. Further details on the experimental procedure can be found elsewhere [12]. High quality $\mathrm{ZnO}$ nanopillars (NPs) grown on a $\mathrm{Si}$ substrate by APMOCVD [13] at $700{ }^{\circ} \mathrm{C}$ were used as a reference sample.

The topography of the obtained nanostructures was studied via scanning electron microscopy (SEM) using a Leo 1550 Gemini SEM operated at voltages ranging from 10 to 20 $\mathrm{kV}$ and using a standard aperture of $30 \mu \mathrm{m}$. The crystal structure of the $\mathrm{ZnO}$ NPs was studied by transmission electron microscopy (TEM). Diffraction contrast images and high resolution images were recorded in a Tecnai G2 FEG electron microscope operated at $200 \mathrm{kV}$. Additionally, the SFs in ZnO NWs were studied using a Carl Zeiss Orion Plus helium-ion microscope (HIM).

The light emission features of the samples were studied using a micro-photoluminescence $(\mu \mathrm{PL})$ spectroscopy setup. The excitation source was a continuous wave UV laser with a wavelength of $266 \mathrm{~nm}$. The laser beam was focused on the samples by a reflective objective to a spot size of about $1.5 \mu \mathrm{m}$ in diameter. The emitted luminescence was collected and mirrored into a TRIAX 550 single grating spectrometer with a focal length of $550 \mathrm{~mm}$, equipped with a 600 grooves/mm grating and a liquid nitrogen cooled CCD camera. The power excitation density was kept constant. The low temperature PL study was performed at $5 \mathrm{~K}$ by helium cooling of a cold-finger type cryostat from Oxford.

Time-resolved $\mu \mathrm{PL}(\mathrm{TR} \mu \mathrm{PL})$ was performed using a Coherent Verdi-V18 (532 nm wavelength) pumped Coherent Mira 900 Ti:Sapphire femtosecond pulsed laser. The output of the Mira 900 was frequency tripled to yield a wavelength of $266 \mathrm{~nm}$. The width of the laser pulses are approximately $200 \mathrm{fs}$, with a repetition rate of $75 \mathrm{MHz}$. The luminescence signal was dispersed by a single grating Chromex 500 is spectrometer with a focal length of $500 \mathrm{~mm}$ and equipped with a 150 grooves $/ \mathrm{mm}$ grating. The time resolved components of the PL were obtained by a Hamamatsu streak camera (model C5680), with a resolution 7 ps. The measurements were done under weak excitation conditions $\left(0.5 \mathrm{~W} / \mathrm{cm}^{2}\right)$. The PL spectra presented in this work were recorded at $5 \mathrm{~K}$. 


\section{Results and discussions}

Figure 1 shows SEM images of the ZnO NWs and the NPs. Both types of nanostructures were of similar length $\sim 1-1.5 \mu \mathrm{m}$. The average diameter of the NWs was $\sim 50 \mathrm{~nm}$ (Fig. 1a). The $\mathrm{ZnO}$ NPs have a telescopic shape: the thicker hexagonal base is transformed into a narrower pillar on top, with an average diameter of $\sim 85 \mathrm{~nm}$. The NWs exhibit fairly uniform diameters, but with notched nanofaceting. Both NWs and NPs samples demonstrate random orientation of the nanostructures, however, NPs has some evidences of axis texture (Fig. 1b). This is because during APMOCVD process NPs are formed as a result of the conventional nucleation of polycrystalline film on the Si substrate, followed by directed growth of the grains along their caxis. The $\mathrm{ZnO} \mathrm{NW}$ sample was in fact a mesh of nanowires, located randomly on the $\mathrm{Si}$ substrate surface. Such morphology is a result of a different growth technique as compared to the growth of the $\mathrm{ZnO}$ NPs $[12,13]$.

A more detailed microstructure analysis was performed by TEM (Fig. 2). It was observed that the ZnO NPs are free of the SFs, but instead a regular arrays of atoms from a perfect crystalline structure, according to high resolution TEM (Fig. 2b). The reason for the telescopic narrowing of the NPs is interpreted as due to a decrease of the precursors' vapor pressures during the growth, as described above.

The NWs exhibit a clear hexagonal structure and around $90 \%$ of them possess corrugated side facets. This confirms the following formation mechanism of the NWs (earlier proposed and described by Glushenkov et al [12]): at the initial stage of growth, ZnO NWs with hexagonal prisms morphology are formed by a fastest growth rate - along the [001] direction. Stacking faults in the NWs naturally introduce steps on the side walls. Under the high precursor vapor supersaturation environment, those stacking faults act as sites of preferential attachment of depositing atoms, leading to observed lateral growth. After the initial transient period, the

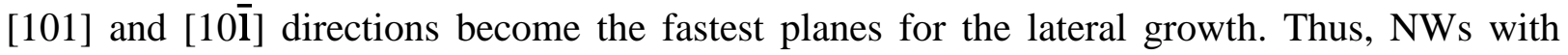
corrugated side facets have been obtained. According to the TEM analysis, the NWs comprise SF located in the (0001) atomic planes with an estimated average density $\sim 1 \times 10^{6} \mathrm{~cm}^{-1}$ as shown in Fig. 2c,d. Inspection of Fig. 2c and d also confirms that the nanofaceting along the wires takes place at the SF intersect with the surface. 
The $\mu \mathrm{PL}$ spectra of the NWs and NPs were found to be different (Fig. 3). The wide range LT PL spectra are different in terms of number of emission peaks and their spectral positions. The LT PL spectrum of the ZnO NPs (Figure 3a) is dominated by the near band edge (NBE) emission, and no deep level (DL) emission was observed. The LT PL spectrum (5 K) of the ZnO NWs with BSF (Figure 3a) comprises both wide and intense DL emission on the lowenergy side and the NBE emission towards the high energy side of the spectrum. The DL emission may be due to point defects (such as oxygen vacancies, zinc interstitials, and their complexes), which could be introduced during growth at high supersaturation.

A detailed analysis of the $\mu \mathrm{PL}$ spectra (Fig. 3b) showed that the NBE emission of ZnO NPs consists of several closely located peaks, at $3.374 \mathrm{eV}, 3.369 \mathrm{eV}$, and $3.365 \mathrm{eV}$, respectively. The highest energy peak $(3.374 \mathrm{eV})$ may be attributed to the surface excitonic emission (SX), while the other two are due to two neutral donor bound excitons $\left(\mathrm{D}^{\circ} \mathrm{X}\right)$.

The analysis of the NBE peaks for ZnO NWs revealed that there is a neutral donor bound exciton peak $\left(\mathrm{D}^{\circ} \mathrm{X}\right)$ at $3.366 \mathrm{eV}$ and another peak at $3.329 \mathrm{eV}$, which is attributed to the photo-emission due to stacking faults (BSF related emission). This is a specific luminescence feature, different from the free or neutral donor bound exciton recombination. Additionally, multiple longitudinal optical phonon (LO) replicas of the present peaks $\left(1^{\text {st }}, 2^{\text {nd }}\right.$ and up to $3^{\text {rd }}$ LO) are separated by $\sim 72 \mathrm{meV}$. Notably, the asymmetry and widths of these observed peaks can be explained by possible overlap with other peaks; the BSF peak may thus be enhanced by two electron satellites (TES) of the $\mathrm{D}^{\circ} \mathrm{X}$ emission, while the observed peak asymmetry may be caused by the $1^{\text {st }}$ LO phonon replica of the $D^{\circ} X$. Similarly, the $2^{\text {nd }}$ LO phonon replica of the $\mathrm{BSF}$ related exciton is unusually strong due to possible overlap with a separate donor-acceptorpair transition.

In order to prove that the two peaks at 3.366 and $3.329 \mathrm{eV}$ are from two different recombination channels, we have investigated their recombination dynamics by TR $\mu \mathrm{PL}$ spectroscopy: the intense $\mathrm{D}^{\circ} \mathrm{X}$ PL peak decays faster, while the emission related to BSFs has a significantly longer decay time (Fig. 4a).

Usually, the PL transient of bulk $\mathrm{ZnO}$ shows a single exponential decay over time, while the $\mathrm{ZnO}$ nanostructures exhibit a non-exponential decay. Excitons recombining at the surface can strongly influence the decay time. Earlier, Zhao et. al. demonstrated that the fast decay component decreases with decreasing of $\mathrm{ZnO}$ nanorod diameter [14]. By examining the decay 
curves obtained for our nanostructures (both NPs and NWs) we have found that the decay curves can be fitted by two exponentials:

$$
I(t)=A_{s} \cdot e^{\frac{-\tau}{\tau_{1}}}+A_{b} \cdot e^{\frac{-\tau}{\tau_{2}}}
$$

where $I(t)$ represents the PL intensity as a function of time, $A_{s}$ and $A_{b}$ are the relative weights of the two exponential functions with the decay constants $\tau_{1}$ and $\tau_{2}$, respectively.

The straight lines in Fig. 4b represent bi-exponential fitted decay curve according to Eq. (1). The results show that for both peaks, the value of $\tau_{1}$ is smaller than $\tau_{2}$ and $\tau_{1}\left(D^{\circ} X\right)=13$ ps and $\tau_{1}(\mathrm{BSF})=19 \mathrm{ps}$. It is believed that the $\tau_{1}$ decay constant represents the surface recombination. For nanostructures, which have large surface area, the surface recombination rate may be dominating, resulting in an observed shorter exciton lifetime [14].

The $\tau_{2}$ values of $\mathrm{D}^{\circ} \mathrm{X}$ and BSF emissions are longer and in addition differ mutually much more: $\tau_{2}\left(\mathrm{D}^{\circ} \mathrm{X}\right)=72 \mathrm{ps}$ and $\tau_{2}(\mathrm{BSF})=360 \mathrm{ps}$, respectively. These time constants are believed to represent exciton decay from the bulk of the NWs. The value of $\tau_{2}\left(\mathrm{D}^{\circ} \mathrm{X}\right)$ agrees well with the earlier reported lifetimes (95 ps) for $\mathrm{ZnO}$ nanostructures of similar size [14].

The large value of $\tau_{2}$ for the BSF related emission can be explained in the following way. As it has been earlier suggested by Rebane et. al. [15], stacking faults in a wurtzite (WZ) $\mathrm{ZnO}$ can be considered as the thinnest segment of the zinc blend (ZB) crystal phase. Since $\mathrm{ZB}$ $\mathrm{ZnO}$ has a smaller band gap than $\mathrm{WZ} \mathrm{ZnO}$, the BSF form a quantum well (QW) like region with negative band offsets in the conduction band minimum and the valence band maximum with respect to those of the wurtzite barrier [16]. The BSF structure acts as a quantum well for electrons and potential barrier for holes in the valence band, with a resulting type II band alignment [17].

Therefore, the peak at 3.329 is interpreted as the recombination of the confined indirect excitons in the BSFs. This transition is due to the recombination of the electrons captured in the quantum wells of the BSF and the holes are bound to the electron at the interface between the $\mathrm{BSF}$ and the wurtzite structure via the attractive Coulomb interaction. Similar phenomena have been reported for the case of $\mathrm{BSF}$ in $\mathrm{GaN}$, InGaN, AlGaN as well as single and multiple QW structures [18]. However, the detailed characteristics of the formed QWs have to be studied by complementary techniques. Nevertheless, we emphasize that the peak that commonly appears in low temperature PL spectra of $\mathrm{ZnO}$ nanostructures at $\sim 3.329 \mathrm{eV}$ is due the presence of basal plane stacking faults in the $\mathrm{ZnO}$ crystal. 


\section{Conclusions}

ZnO NWs with high concentrations of BSFs demonstrate a PL peak at $\sim 3.329 \mathrm{eV}$ (at 5 $\mathrm{K})$. The peak appears along with the commonly observed $\mathrm{D}^{\circ} \mathrm{X}$ peak and is followed by its LO phonon replicas. Transient PL characteristics of both peaks can be fitted by bi-exponential models with short $\tau_{1}$ and long $\tau_{2}$ decay constants. The shorter decay constants for both the BSF and the $\mathrm{D}^{\circ} \mathrm{X}$ PL peaks represent the fast surface recombination due to surface states trapping the excitons. The $\tau_{2}$ component of the decay curve for the PL peak of the $\mathrm{D}^{\circ} \mathrm{X}$ emission was estimated to be $72 \mathrm{ps}$, while the $\tau_{2}$ decay constant of the BSF related PL peak is $360 \mathrm{ps}$. The suggested luminescence mechanism of this peak is an indirect exciton originating from the recombination of electrons in the QW (ZB) and holes in the WZ crystal localized near a BSF.

\section{Acknowledgements}

Swedish Research Link (SRL-VR) 2009-6427 “ZnO Nano Structures for Energy Saving Light Emitters" (2010 - 2012) and the Linköping Linnaeus initiative for Novel Functional Materials (LiLi-NFM) Linné Grant at IFM LiU are acknowledged for the support of this work. L. Hultman and G.Z. Radnoczi acknowledge the Knut and Alice Wallenberg Foundation for supporting the Electron Microscopy Laboratory operated by the Thin Film Physics Division of Linköping University. Authors would like to thank Dr Alexey Glushenkov and Prof Ying Chen from Deakin University, Australia, for providing a sample of $\mathrm{ZnO}$ nanowires. Prof P. Bergman from Linköping University is acknowledged for the fruitful discussions.

\section{References}

[1] Ü. Özgür, Ya. I. Alivov, C. Liu, A. Teke, M. A. Reshchikov, S. Doğan, V. Avrutin, S.-J. Cho, and H. Morkoç, J. Appl. Phys. 98 (2005) 041301

[2] V. Khranovskyy, G. R. Yazdi, G. Lashkarev, A. Ulyashin, and R. Yakimova, Phys. Stat. Sol. (a) 205 (2008) 144

[3] V. Khranovskyy, I. Tsiaoussis, G. R. Yazdi, L. Hultman and R. Yakimova, Journal of Crystal Growth, 312 (2010) 327 
[4] V. Karpyna, A. Evtukh, M. Semenenko, V. Lazorenko, G. Lashkarev, V. Khranovskyy, R. Yakimova, D. Fedorchenko, Microelectronics Journal 40 (2009) 229

[5] A. Ievtushenko, G. Lashkarev, V. Lazorenko, V. Karpyna, V. Sichkovskyj, L. Kosyachenko, V. Sklyarchuk, O. Sklyarchuk, V. Bosy, F. Korzhinski, A. Ulyashin, V. Khranovskyy and R. Yakimova, Acta Physica Polonica A 114 (2008) 1123

[6] D. Spirkoska, J. Arbiol, A. Gustafsson, S. Conesa-Boj, et al , Phys. Rev. B 80 (2009) 245325

[7] V. Khranovskyy, I. Tsiaoussis, L. Hultman and R. Yakimova, Nanotechnology 22 (2011) 185603

[8] A. Djurisic, A. Ng, X. Chen, Progress in Quantum Electronics 34 (2010) 191

[9] Paskov P P, Schifano R, Monemar B, Paskova T, Figge S and Hommel D, J. Appl. Phys. 98 (2005) 093519

[10] Thonke K, Schirra M, Schneider R, Reiser A, Prinz G M, Feneberg M, Biskupek J, Kaiser U, Sauer R, Microelectronics J 40 (2009) 210

[11] Thonke K, Schirra M, Schneider R, Reiser A, et al. Physica Status Solidi B 247 (2010) 1464

[12] A. M. Glushenkov, H. Zhang, J. Zou, G. Qing Lu, Y. Chen, J. Cryst. Growth 310 (2008) 3139

[13] V. Khranovskyy and R. Yakimova, Physica B: Condenced Matter 407 (2012) 1538

[14] Q. X. Zhang, L. L. Yang, M. Willander, B. E. Sernelius, P. O. Holtz J. Appl. Phys. 104 (2008) 073526

[15] Y. T. Rebane, Y. G. Shreter and A. M. Albrecht, Phys. Stat. Solidi (a) 164 (1997) 141

[16] J. Lähnemann, O. Brandt, U. Jahn, C. Pfuller, C. Roder, P. Dogan, F. Grosse, A. Belabbes, F. Bechstedt, A. Trampert and Geelhaar Phys. Rev. B 86 (2012) 081302(R)

[17] Y. Yan, G. M. Dalpian, M. M. Al-Jassim and S-.H. Wei, Phys. Rev. B 70 (2004) 193206 
[18] K. S. Ramaiah, Y. K. Su, S. J. Chang, B. Kerr, H. P. Liu I. G. Chen, Appl. Phys. Lett. 84 (2004) 3307

\section{Figure captions}

Figure 1. a) Top view SEM images of the investigated $\mathrm{ZnO}$ (a) NWs and (b) NPs.

Figure 2. Detailed topography of $\mathrm{ZnO}$ nanostructures: (a) TEM bright field and (b) HRTEM images of defect free ZnO NPs (tip region of the NPs is shown); and (c) TEM overview image of $\mathrm{ZnO} \mathrm{NW}$ : the dark lines perpendicular to the NW length and corresponding to BSFs show defect distribution along the NW; and (d) dark field TEM image of BSFs in individual ZnO NW.

Figure 3. Low temperature (5 K) PL spectra of ZnO NWs, containing BSFs vs. defect-free NPs; (a) wide range PL spectrum of NWs comprising both defect emission and near band edge (NBE) emission, while NPs demonstrate only NBE emission; and (b) detailed NBE emission of ZnO NWs vs. NPs.

Figure 4. TR $\mu \mathrm{PL}$ image of $\mathrm{ZnO}$ NWs (a) two peaks with different PL lifetimes; (b) transient PL characteristics of $\mathrm{D}^{\circ} \mathrm{X}$ and $\mathrm{BSF}$ related exciton emissions, respectively. 


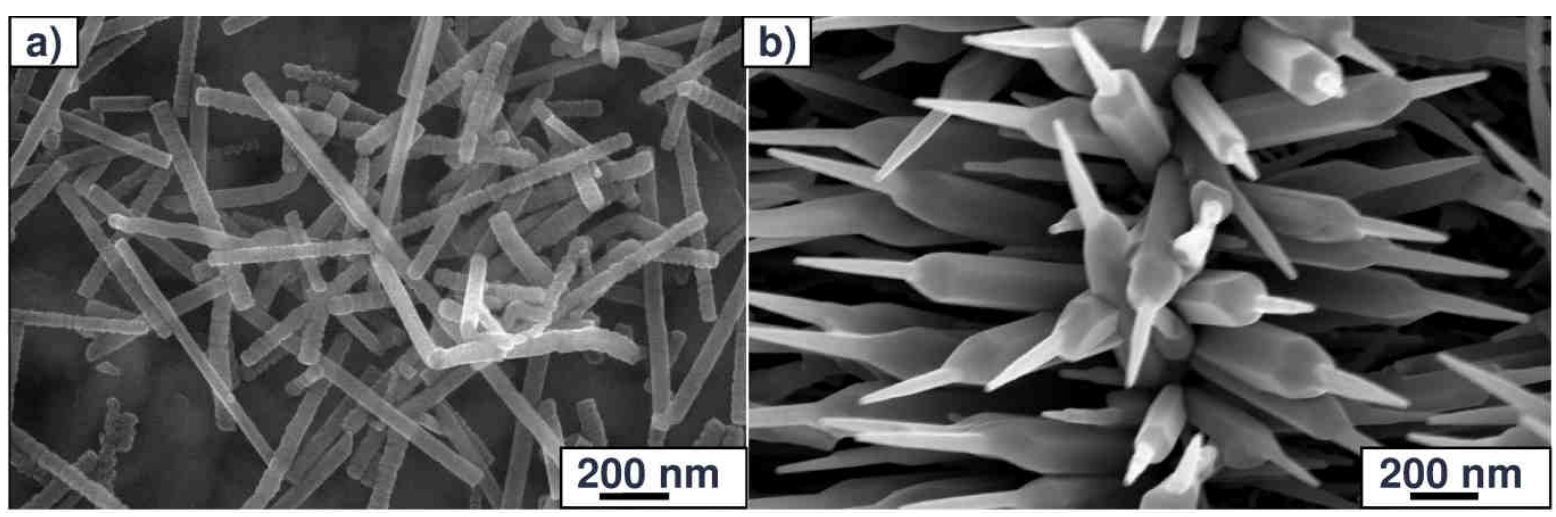

Figure 1. 

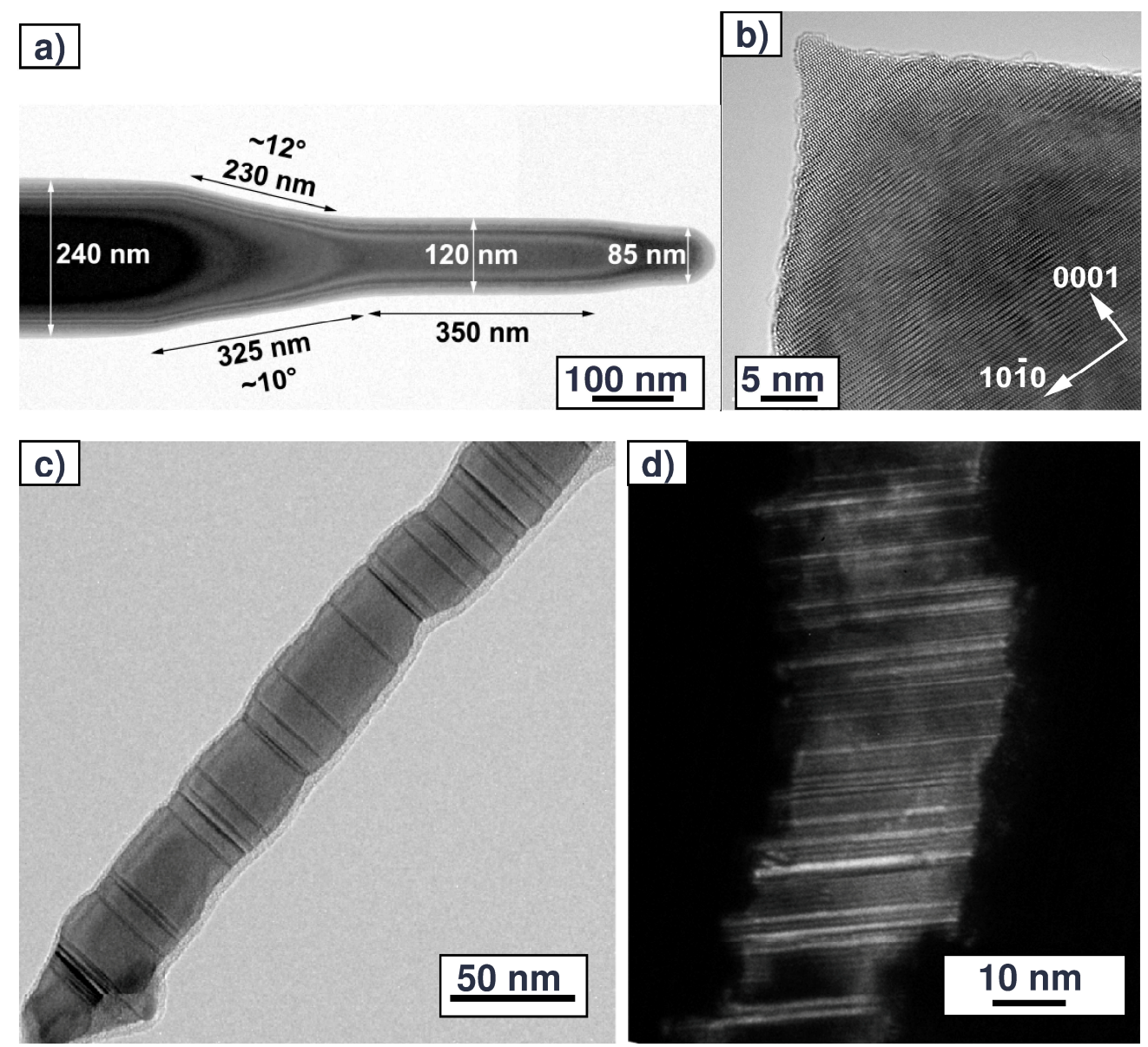

Figure 2. 

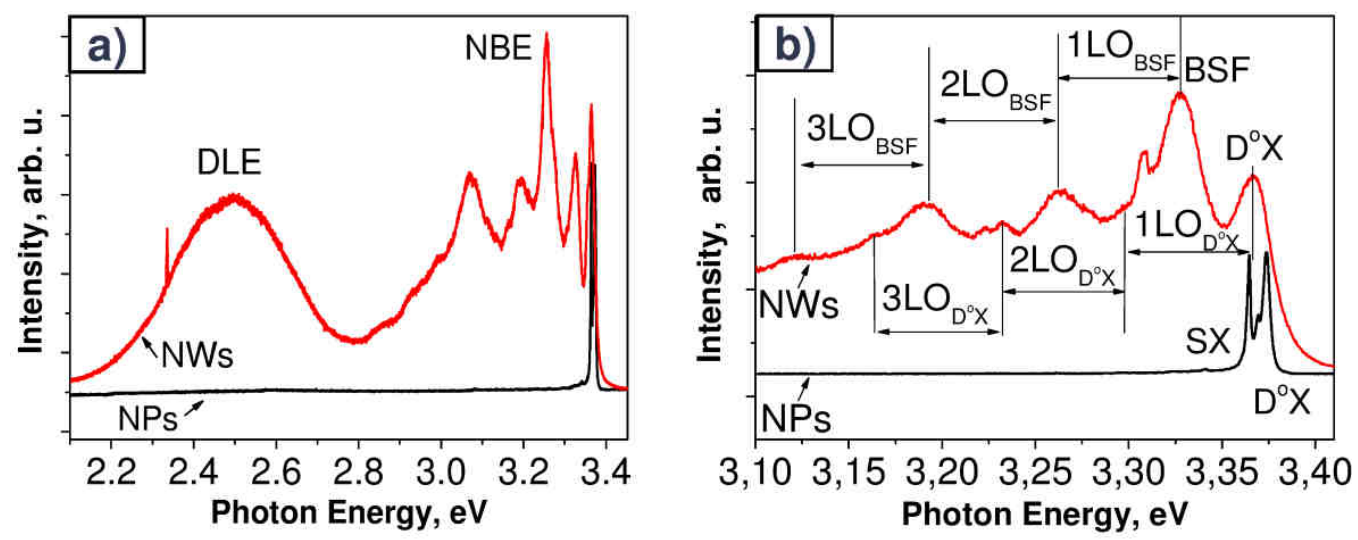

Figure 3. 

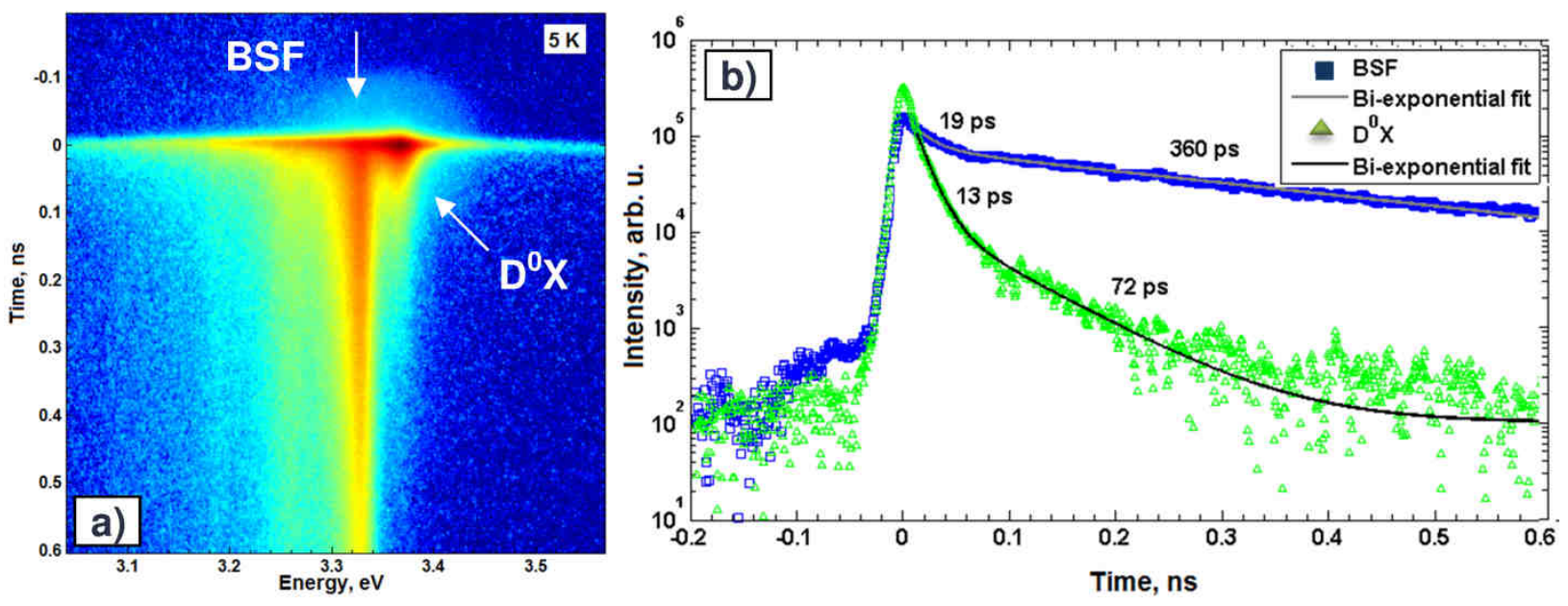

Figure 4. 